\title{
Utilizing the Most Accurate Preoperative Risk Calculator
}

\author{
Wasey Ali Yadullahi Mir ${ }^{1}$, Francesco Fiumara ${ }^{2}$, Dhan B. Shrestha ${ }^{1}$, Suman Gaire ${ }^{3}$, Larissa Verda ${ }^{1}$ \\ 1. Department of Internal Medicine, Mount Sinai Hospital, Chicago, USA 2. Department of Internal Medicine, \\ University of Miami Palm Beach, Atlantis, USA 3. Department of Emergency Medicine, Palpa Hospital, Palpa, NPL
}

Corresponding author: Wasey Ali Yadullahi Mir, drmirwaseyali@gmail.com

\begin{abstract}
The most commonly used preoperative assessment tools include the American College of Surgeons National Surgical Quality Improvement Program and the Revised Cardiac Risk Index. These tools seek to predict the risk of an individual experiencing postoperative complications, including but not limited to mortality, myocardial infarction, pneumonia, stroke, venous thromboembolism, and pneumonia. Many published studies have sought to objectively quantify the utility of the preoperative risk calculations by retrospectively compiling data for patients who underwent the same or comparable surgeries to compare actual complications to predicted complications. Therefore, we searched these studies to review the literature to draw more general conclusions and recommend which risk calculator is best for different types of surgeries.
\end{abstract}

Review began $07 / 26 / 2021$ Review ended 08/03/2021 Published 08/10/2021

๑) Copyright 2021 Mir et al. This is an open access article distributed under the terms of the Creative Commons Attribution License CC-BY 4.0., which permits unrestricted use, distribution, and reproduction in any medium, provided the original author and source are credited.
Categories: Cardiac/Thoracic/Vascular Surgery, General Surgery

Keywords: quality improvement, venous thromboembolism, myocardial infarction, postoperative complications, surgeons

\section{Introduction And Background}

Patients undergoing major noncardiac surgery are also at an increased risk of perioperative cardiovascular events. The risk depends both on the nature of the noncardiac surgery and the patient's health status. In a study that included more than 10 million hospitalizations for major noncardiac surgery, the incidence of a major adverse cardiovascular and cerebrovascular event was found to be $3 \%$ [1]. Therefore, all patients undergoing noncardiac surgery should be assessed for the risk of perioperative cardiovascular events and measures should be taken for prevention, if necessary. There are multiple tools available for the assessment. Two of the most commonly used tools are the Revised Cardiac Risk Index (RCRI) and the American College of Surgeons National Surgical Quality Improvement Program (ACS-NSQIP).

RCRI consists of the following six independent predictors of major cardiac complications: a high-risk surgery, history of ischemic heart disease, history of heart failure, cerebrovascular disease, diabetes mellitus requiring insulin, and preoperative serum creatinine $>2.0 \mathrm{mg} / \mathrm{dL}$. In addition, the risk of cardiac complications is estimated based on the number of risk factors present [2]. RCRI was initially validated in a cohort of 1,422 individuals and has been subsequently evaluated by other studies [2,3].

The ACS-NSQIP consists of the following five perioperative myocardial infarction or cardiac arrest predictors: the type of surgery, dependent functional status, abnormal serum creatinine, age, and the American Society of Anesthesiologists class [4].

In this study, we reviewed 16 case series in detail that assessed the utility of these risk assessments by querying the PubMed database. The validity of the conclusions was evaluated by analyzing the results as a whole. Both indices were reviewed for intra-abdominal, vascular, transplant, neurosurgeries, and noncardiac surgeries. We sought to examine the conclusions of each statistical measure to determine whether the outcome for that risk calculator was predictable, not predictable, or unpredictable.

\section{Review}

\section{National Surgical Quality Improvement Program}

Intra-abdominal Surgery

The NSQIP was not reliable for predicting outcomes. For open abdominal aortic aneurysm repair and endovascular aneurysm repair, predictability was 0.51 and 0.02 , respectively. The predictability for open or robotic radical cystectomy using the NSQIP calculator was neither effective nor better than existing models $[5]$.

Vascular Surgery 
The NSQIP was ineffective for predicting the postoperative outcomes of vascular surgeries. In addition, the NSQIP was unreliable for predicting outcomes in both carotid endarterectomies and infra-inguinal lower extremity bypasses, showing predictability of 0.45 and 0.054 , respectively [6].

\section{Transplant Surgery}

Canbolat et al. utilized the NSQIP in patients undergoing living donor liver transplantation and concluded no correlation between the NSQIP and the postoperative outcome of adverse events [7].

\section{Neurosurgery}

Although not perfect, the NSQIP is a better risk calculator for neurosurgeries. It was described as not predictive in geriatric patients undergoing lumbar surgery, with a Brier score of 0.321. Notwithstanding, the NSQIP proved to be accurate for predicting adverse outcomes in patients undergoing elective craniotomies for the small sample size in the study by Rutowski et al. [8]. Despite the low power of the former study, the NSQIP was described by Veeravagu et al. to be effective for predicting adverse outcomes in patients undergoing spinal surgery, highlighting an area under the curve (AUC) of 0.669 in larger sample size [9].

\section{Biomarkers in Noncardiac Surgery}

One study utilized the NSQIP to assess patients undergoing noncardiac surgery. Markovic et al. used the area under the receiver operating characteristic curve to analyze whether the NSQIP could accurately predict outcomes when compared with the biomarkers baculoviral IAP repeat-containing protein 5, heart-type fatty acid-binding protein, and high sensitivity C-reactive protein. The results showed an AUC of 0.941 when combining the NSQIP with the three biomarkers, indicating excellent predictability [10].

\section{Revised Cardiac Risk Index}

Intra-abdominal Surgery

The RCRI demonstrated mixed results for predicting outcomes. It did not reliably predict postoperative outcomes in either open abdominal aortic aneurysm repairs or endovascular aneurysm repairs [6]. However, it was reliable for predicting adverse outcomes in the studies describing major digestive, breast, or vascular surgery in patients $>65$ years old [11] and endovascular abdominal aortic aneurysm repair [12].

Vascular Surgery

The RCRI overall proved to be ineffective for predicting outcomes. It was unreliable when predicting outcomes in both carotid endarterectomies and infra-inguinal lower extremity bypasses, showing predictability of 0.17 and 0.002 , respectively. Although the statistical measure showed some positive association using the AUC, in three of the five studies, the RCRI was determined to be unreliable. RCRI demonstrated predictability in noncardiac vascular surgery (odds ratio $[\mathrm{OR}]=3.9)$ and vascular surgery $(\mathrm{OR}=$ 1.73) $[13,14]$. Both of these studies used OR as the statistical measure of choice. It should be considered that if the three studies describing AUC results of $<0.70$ and $>0.55$ utilized OR as well, there may have been significant findings.

Transplant Surgery

According to a study, the RCRI was not predictable, describing a weakly positive correlation of 0.245 . Conversely, it was reliable and successful in predicting adverse outcomes in both liver and kidney transplants. Park et al. measured adjusted OR to be 4.35, indicating a positive result for RCRI patients who underwent liver transplantation [15]. Hoftman et al. described an AUC of 0.77 for patients undergoing kidney transplantation [16].

Neurosurgery

The RCRI was found to be predictive for patients undergoing elective craniotomies, as described by Rutkowski et al., utilizing a small sample study [8]. However, Carabini et al., utilizing a significantly larger study sample, reported the RCRI to be unreliable for predicting adverse outcomes in patients undergoing spinal fusion with instrumentation, reporting an AUC of 0.54 [17].

Biomarkers in Noncardiac Surgery

RCRI was found to have resoundingly positive predictability when combined with specific biomarkers. The adjusted hazard ratio demonstrated predictability in the RCRI combined with N-terminal pro-B-type natriuretic peptide for noncardiac surgeries [18]. Kopec et al. utilized high-sensitivity cardiac troponin T (hscTnT) combined with the RCRI, resulting in an AUC of 0.716 for patients undergoing major noncardiac 


\section{Cureus}

surgery. Interestingly, in the same study, when the RCRI was used on its own, without hs-cTnT, the AUC was found to be 0.59 , a result that indicated unpredictability [19]. Lastly, Golubovic et al. utilized high-sensitivity troponin I in combination with the RCRI for patients undergoing major elective vascular surgery in which the AUC was $0.909[20]$.

Based on the review of existing literature and objective analysis, the NSQIP fell short in reliably predicting postoperative complications for the majority of surgeries. Conversely, the RCRI was more reliable in accurately predicting postoperative complications. The best preoperative risk calculator recommendations for each type of surgery are outlined in Table 1 .

\begin{tabular}{|l|l|}
\hline Category of surgery & Risk calculator \\
\hline Intra-abdominal & RCRI \\
\hline Vascular & RCRI \\
\hline Transplant & RCRI \\
\hline Neurosurgery & NSQIP \\
\hline Noncardiac with biomarkers & Either or both \\
\hline
\end{tabular}

TABLE 1: Category of surgery and the appropriate risk calculator.

RCRI: Revised Cardiac Risk Index; NSQIP: National Surgical Quality Improvement Program

\section{Conclusions}

The RCRI was determined to be the more effective risk calculator in predicting postoperative outcomes for intra-abdominal, vascular, and transplant surgeries. The NSQIP was determined to be the more effective risk calculator in predicting the postoperative outcomes for neurosurgical surgeries. In the case of noncardiac surgery, when supplemented with biomarkers, both the RCRI and NSQIP proved to be highly effective at predicting outcomes.

\section{Additional Information}

\section{Disclosures}

Conflicts of interest: In compliance with the ICMJE uniform disclosure form, all authors declare the following: Payment/services info: All authors have declared that no financial support was received from any organization for the submitted work. Financial relationships: All authors have declared that they have no financial relationships at present or within the previous three years with any organizations that might have an interest in the submitted work. Other relationships: All authors have declared that there are no other relationships or activities that could appear to have influenced the submitted work.

\section{References}

1. Smilowitz NR, Gupta N, Ramakrishna H, Guo Y, Berger JS, Bangalore S: Perioperative major adverse cardiovascular and cerebrovascular events associated with noncardiac surgery. JAMA Cardiol. 2017, 2:181-7. 10.1001/jamacardio.2016.4792

2. Lee TH, Marcantonio ER, Mangione CM, et al.: Derivation and prospective validation of a simple index for prediction of cardiac risk of major noncardiac surgery. Circulation. 1999, 100:1043-9. 10.1161/01.cir.100.10.1043

3. Devereaux PJ, Goldman L, Cook DJ, Gilbert K, Leslie K, Guyatt GH: Perioperative cardiac events in patients undergoing noncardiac surgery: a review of the magnitude of the problem, the pathophysiology of the events and methods to estimate and communicate risk. CMAJ. 2005, 173:627-34. 10.1503/cmaj.050011

4. Gupta PK, Gupta H, Sundaram A, et al.: Development and validation of a risk calculator for prediction of cardiac risk after surgery. Circulation. 2011, 124:381-7. 10.1161/CIRCULATIONAHA.110.015701

5. Mannas MP, Lee T, Forbes CM, et al.: Predicting complications following radical cystectomy with the ACS NSQIP universal surgical risk calculator. World J Urol. 2020, 38:1215-20. 10.1007/s00345-019-02915-3

6. Moses DA, Johnston LE, Tracci MC, Robinson WP 3rd, Cherry KJ, Kern JA, Upchurch GR Jr: Estimating risk of adverse cardiac event after vascular surgery using currently available online calculators. J Vasc Surg. 2018, 67:272-8. 10.1016/j.jvs.2017.06.105

7. Canbolat IP, Erdogan Y, Adali G, Kaplan O, Dayangac M, Yuzer Y, Tokat Y: The predictive value of risk indices for cardiac complications in living donor liver transplantation. Bratisl Lek Listy. 2018, 119:289-93. 10.4149/BLL 2018054

8. Rutkowski M, Sankaran S: Preoperative risk stratification of patient mortality following elective craniotomy; a comparative analysis of prediction algorithms. J Clin Neurosci. 2019, 67:24-31. 10.1016/j.jocn.2019.06.037 
9. Veeravagu A, Li A, Swinney C, et al.: Predicting complication risk in spine surgery: a prospective analysis of a novel risk assessment tool. J Neurosurg Spine. 2017, 27:81-91. 10.3171/2016.12.SPINE16969

10. Markovic DZ, Jevtovic-Stoimenov T, Stojanovic M, Vukovic AZ, Dinic V, Markovic-Zivkovic BZ, Jankovic RJ: Cardiac biomarkers improve prediction performance of the combination of American Society of Anesthesiologists physical status classification and Americal College of Surgeons National Surgical Quality Improvement Program calculator for postoperative mortality in elderly patients: a pilot study. Aging Clin Exp Res. 2019, 31:1207-17. 10.1007/s40520-018-1072-0

11. Hirano Y, Takeuchi H, Suda K, et al.: Clinical utility of the Revised Cardiac Risk Index in non-cardiac surgery for elderly patients: a prospective cohort study. Surg Today. 2014, 44:277-84. 10.1007/s00595-013-0543-3

12. Archan S, Roscher CR, Fairman RM, Fleisher LA: Revised Cardiac Risk Index (Lee) and perioperative cardiac events as predictors of long-term mortality in patients undergoing endovascular abdominal aortic aneurysm repair. J Cardiothorac Vasc Anesth. 2010, 24:84-90. 10.1053/j.jvca.2009.04.003

13. Yang JH, Choi JH, Ki YW, et al.: Plasma N-terminal pro-B-type natriuretic peptide is predictive of perioperative cardiac events in patients undergoing vascular surgery. Korean J Intern Med. 2012, 27:301-10. 10.3904/kjim.2012.27.3.301

14. Garcia S, Moritz TE, Goldman S, et al.: Perioperative complications after vascular surgery are predicted by the revised cardiac risk index but are not reduced in high-risk subsets with preoperative revascularization. Circ Cardiovasc Qual Outcomes. 2009, 2:73-7. 10.1161/CIRCOUTCOMES.108.827683

15. Park YS, Moon YJ, Jun IG, Song JG, Hwang GS: Application of the Revised Cardiac Risk Index to the Model for End-Stage Liver Disease Score improves the prediction of cardiac events in patients undergoing liver transplantation. Transplant Proc. 2018, 50:1108-13. 10.1016/j.transproceed.2018.01.024

16. Hoftman N, Prunean A, Dhillon A, Danovitch GM, Lee MS, Gritsch HA: Revised Cardiac Risk Index (RCRI) is a useful tool for evaluation of perioperative cardiac morbidity in kidney transplant recipients. Transplantation. 2013, 96:639-43. 10.1097/TP.0b013e31829e2703

17. Carabini LM, Zeeni C, Moreland NC, et al.: Predicting major adverse cardiac events in spine fusion patients: is the revised cardiac risk index sufficient?. Spine (Phila Pa 1976). 2014, 39:1441-8. 10.1097/BRS.0000000000000405

18. Duceppe E, Patel A, Chan MT, et al.: Preoperative N-terminal pro-B-type natriuretic peptide and cardiovascular events after noncardiac surgery: a cohort study. Ann Intern Med. 2020, 172:96-104. 10.7326/M19-2501

19. Kopec M, Duma A, Helwani MA, et al.: Improving prediction of postoperative myocardial infarction with high-sensitivity cardiac troponin T and NT-proBNP. Anesth Analg. 2017, 124:398-405. 10.1213/ANE.0000000000001736

20. Golubovic M, Stanojevic D, Lazarevic M, et al.: A risk stratification model for cardiovascular complications during the 3-month period after major elective vascular surgery. Biomed Res Int. 2018, 2018:4381527. $10.1155 / 2018 / 4381527$ 